\title{
Optimizing minimum free-energy crossing points in solution: Linear- response free energy/spin-flip density functional theory approach.
}

\author{
$\operatorname{AUTHOR}(S)$ :
}

Minezawa, Noriyuki

\section{CITATION:}

Minezawa, Noriyuki. Optimizing minimum free-energy crossing points in solution: Linearresponse free energy/spin-flip density functional theory approach.. The Journal of chemical physics 2014, 141(16): 164118.

\section{ISSUE DATE:}

2014-10-28

URL:

http://hdl.handle.net/2433/191226

\section{RIGHT:}

Copyright 2014 American Institute of Physics. This article may be downloaded for personal use only. Any other use requires prior permission of the author and the American Institute of Physics. 




\section{Optimizing minimum free-energy crossing points in solution: Linear-response free energy/spin-flip density functional theory approach}

Noriyuki Minezawa

Citation: The Journal of Chemical Physics 141, 164118 (2014); doi: 10.1063/1.4899049

View online: http://dx.doi.org/10.1063/1.4899049

View Table of Contents: http://scitation.aip.org/content/aip/journal/jcp/141/16?ver=pdfcov

Published by the AIP Publishing

\section{Articles you may be interested in}

Excited-state free energy surfaces in solution: Time-dependent density functional theory/reference interaction site model self-consistent field method

J. Chem. Phys. 138, 244101 (2013); 10.1063/1.4811201

Valence excitation energies of alkenes, carbonyl compounds, and azabenzenes by time-dependent density functional theory: Linear response of the ground state compared to collinear and noncollinear spin-flip TDDFT with the Tamm-Dancoff approximation

J. Chem. Phys. 138, 134111 (2013); 10.1063/1.4798402

Free energy decomposition analysis of bonding and nonbonding interactions in solution

J. Chem. Phys. 137, 034111 (2012); 10.1063/1.4736533

Optimizing conical intersections of solvated molecules: The combined spin-flip density functional theory/effective fragment potential method

J. Chem. Phys. 137, 034116 (2012); 10.1063/1.4734314

Complete structural and magnetic characterization of biological radicals in solution by an integrated quantum mechanical approach: Glycyl radical as a case study

J. Chem. Phys. 121, 6710 (2004); 10.1063/1.1791031


\section{SUBSCRIBE TO \\ physjcs today}




\title{
Optimizing minimum free-energy crossing points in solution: Linear-response free energy/spin-flip density functional theory approach
}

\author{
Noriyuki Minezawa ${ }^{\text {a) }}$ \\ Fukui Institute for Fundamental Chemistry, Kyoto University, Sakyo-ku, Kyoto 606-8103, Japan
}

(Received 7 August 2014; accepted 10 October 2014; published online 28 October 2014)

\begin{abstract}
Examining photochemical processes in solution requires understanding the solvent effects on the potential energy profiles near conical intersections (CIs). For that purpose, the CI point in solution is determined as the crossing between nonequilibrium free energy surfaces. In this work, the nonequilibrium free energy is described using the combined method of linear-response free energy and collinear spin-flip time-dependent density functional theory. The proposed approach reveals the solvent effects on the CI geometries of stilbene in an acetonitrile solution and those of thymine in water. Polar acetonitrile decreases the energy difference between the twisted minimum and twistedpyramidalized CI of stilbene. For thymine in water, the hydrogen bond formation stabilizes significantly the CI puckered at the carbonyl carbon atom. The result is consistent with the recent simulation showing that the reaction path via this geometry is open in water. Therefore, the present method is a promising way of identifying the free-energy crossing points that play an essential role in photochemistry of solvated molecules. (C) 2014 AIP Publishing LLC. [http://dx.doi.org/10.1063/1.4899049]
\end{abstract}

\section{INTRODUCTION}

Conical intersections (CIs) ${ }^{1-4}$ play a fundamental role in photochemical and photobiological processes of molecules in electronically excited states. The CI seam forms a funnel connecting multiple electronic states and provides efficient relaxation pathways. The environment changes the mechanism of photochemistry. Polar solvents stabilize the ionic state more efficiently than the nonpolar covalent state. The location of crossing seam under the polar environment is different from that estimated under the isolated condition. The importance of solvent effects on the CI structure and nonadiabatic transitions has been pointed out for several molecules. ${ }^{5}$ For example, the purine and pyrimidine bases, which are building blocks of DNA and RNA, show qualitatively different relaxation mechanisms in solution. ${ }^{6,7}$ Another interesting issue is controlling the photochemical reaction by solvent polarity. The solvent can modulate the excited-state lifetime because the location of CI point depends critically on the solute-solvent interaction. The recent studies suggest that the solvent tunes CI points and that the external electric field or solvent polarity controls competing relaxation pathways and branching ratio. ${ }^{8-10}$

Nonadiabatic simulation incorporating the solvent effect is an ideal tool that gives valuable insights into the mechanisms. Moreover, one can compare the results such as lifetime and product branching ratio to experimental results directly. Running many trajectories, however, is not feasible because describing excited-state electronic structure and CIs requires accurate methods taking account of both static and dynamic electron correlation effects. Thus, some "static" method is a useful alternative to describe the CI points in solution. Toniolo et al. ${ }^{11}$ discuss the solvent effects on the potential energy surface (PES) crossing by using the quantum

\footnotetext{
${ }^{a)}$ E-mail: minezawa@ fukui.kyoto-u.ac.jp
}

mechanics/molecular mechanics $(\mathrm{QM} / \mathrm{MM})$ method at the semiempirical configuration-interaction level. Because the solvent relaxation after photoexcitation is out of equilibrium, the description of the nonequilibrium solvation needs some solvation coordinate measuring the deviation from the equilibrium. Hynes and co-workers ${ }^{12-16}$ considered the nonequilibrium free energy within the dielectric continuum model by introducing the orientational polarization vector as an effective solvation coordinate. These authors examined the nonequilibrium solvation effects on the topology of $\mathrm{CI}$ for model protonated Schiff base (PSB) and found that the increase in solvation coordinate converts the sloped CI structure to a peaked CI. Losa et al. ${ }^{17,18}$ found the free-energy crossing points of acrolein in water by using the averaged solvent electrostatic potential method and examined the solvent effect on the internal conversion and intersystem crossing. Cui and Yang ${ }^{19}$ derived the scheme of optimizing CI points in solution by the QM/MM molecular dynamics (MD) simulation. They performed the QM CI optimization and the MM minimization (or sampling configurations) sequentially and identified successfully several CI points in solution. Yamazaki and Kato $^{20,21}$ developed the linear-response free energy (LRFE) method and optimized the minimum free-energy crossing points of ethylene, $\mathrm{CH}_{2} \mathrm{NH}_{2}{ }^{+}$, and $9 \mathrm{H}$-adenine. Later, Mori et $a .^{22}$ reformulated the LRFE method and determined the crossing points of PSB in methanol solution at the multireference perturbation theory level.

The LRFE approach ${ }^{20-25}$ introduces the electrostatic potential (ESP) acting on solute atomic sites as the solvation coordinate mimicking solvent orientational polarization. That approach assumes the Gaussian probability distribution of the ESP around some reference-state ESP. ${ }^{26,27}$ The previous studies describe the reference state by either the QM/MM MD simulation ${ }^{23}$ or the reference interaction site model self-consistent field (RISM-SCF) method. ${ }^{28-30}$ The resultant 
(a)

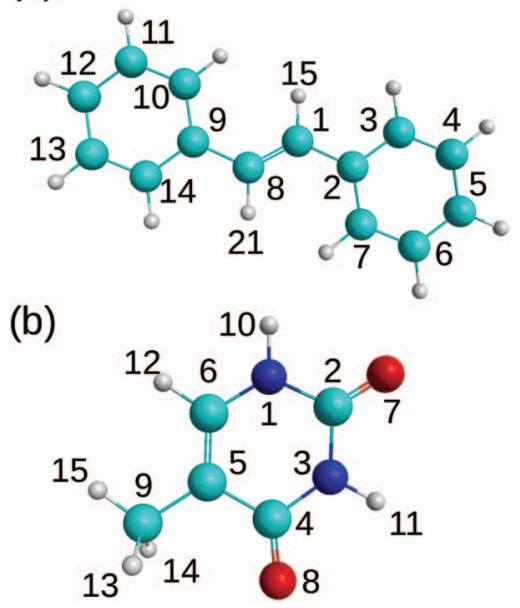

FIG. 1. Molecular structures of (a) stilbene and (b) thymine: carbon (cyan), nitrogen (blue), oxygen (red), and hydrogen (white) atoms.

nonequilibrium free energy is dependent on the solvation coordinate as well, and it is necessary to optimize both the solute and solvation coordinates in finding equilibrium minimum and crossing points. In the present work, the collinear spinflip time-dependent density functional theory (SF-TDDFT or simply SFDFT) $)^{31-37}$ is combined with the LRFE method to locate the free-energy crossing points of solvated molecules. In contrast to the conventional TDDFT, the SFDFT uses the triplet state with two unpaired alpha electrons as reference and takes the alpha-to-beta spin-flip excitations to generate target states. Thus, the method can describe the ground and first-excited states on an equal footing. The electronic structure methods based on spin-flip excitations have recently been used successfully to describe the CI points in vacuum. ${ }^{38-49} \mathrm{In}$ this work, the hybrid method of LRFE and SFDFT is developed to evaluate the minimum energy crossing point (MECP) in solution. The solvent effects are examined for (1) the photoisomerization reaction of stilbene in an acetonitrile solution and (2) the nonadiabatic processes of thymine in water (see Fig. 1).

The organization of this paper is as follows. Section II describes the formulation of the LRFE-SFDFT energy and gradient. The algorithm locating the MECP is modified to take account of solvation coordinate. In Sec. III, the proposed method is applied to study the solvent effects on the $\mathrm{S}_{0} / \pi \pi^{*}$ crossing points of stilbene and thymine. Concluding remarks are presented in Sec. IV.

\section{THEORETICAL METHOD}

\section{A. SFDFT method for nonequilibrium free energy}

The author has recently developed a hybrid method of LRFE $^{22}$ and conventional TDDFT and discussed the state-specific solvation effect on the intramolecular charge transfer. ${ }^{50}$ The LRFE-SFDFT method is a simple extension of that work. The nonequilibrium free energy for electronically excited states is given as a function of not only nuclear coordinate $\mathbf{R}$ but also solvation coordinate $\mathbf{V}$ that consists of the ESP acting on solute atomic sites,

$$
\begin{aligned}
G_{\text {noneq }}(\mathbf{R}, \mathbf{V})= & E_{\text {elec }}(\mathbf{R}, \mathbf{V})-\mathbf{V}^{T} \mathbf{Q}_{0}+\Delta \mu_{0} \\
& +\frac{1}{2 \beta}\left(\mathbf{V}-\mathbf{V}_{0}\right)^{T} \mathbf{C}_{0}^{-1}\left(\mathbf{V}-\mathbf{V}_{0}\right) .
\end{aligned}
$$

$\mathbf{Q}_{0}, \mathbf{V}_{0}$, and $\Delta \mu_{0}$ are the partial charges, the solvation coordinate, and the excess chemical potential for the reference state, respectively, and are determined by the reference-state RISM-SCF calculation. ${ }^{28-30}$ Note that $\mathbf{V}_{0}$ is the equilibrium solvation coordinate for the reference state. The solvation coordinate introduced in this work represents the slow orientational polarization (fast electronic polarization is not considered in this work) because $\mathbf{V}_{0}$ reflects the average solvent position, i.e., radial distribution functions. The last term defines the solvent fluctuation around the reference-state equilibrium solvation structure and assumes that the probability of finding the solvation coordinate $\mathbf{V}$ is given by the multi-dimensional Gaussian. ${ }^{26,27}$ The matrix $\mathbf{C}_{0}$ is the ESP covariance,

$$
\mathbf{C}_{0} \equiv\left\langle\left(\mathbf{V}-\mathbf{V}_{0}\right)\left(\mathbf{V}-\mathbf{V}_{0}\right)^{T}\right\rangle_{0}=-\beta^{-1} \frac{\partial \Delta \mu_{0}}{\partial \mathbf{Q}_{0} \partial \mathbf{Q}_{0}}=-\beta^{-1} \frac{\partial \mathbf{V}_{0}}{\partial \mathbf{Q}_{0}},
$$

where $\beta$ is the inverse temperature. The ESP derivative $\partial \mathbf{V}_{0} / \partial \mathbf{Q}_{0}$ is obtained by solving the first-order coupledperturbed RISM equations. $^{20,27}$

The solute electronic energy $E_{\text {elec }}(\mathbf{R}, \mathbf{V})$ in Eq. (1) is computed under the external ESP $\mathbf{V}$ and given by the sum of Kohn-Sham energy functional $E_{\mathrm{KS}}(\mathbf{R}, \mathbf{V})$ and collinear SFDFT transition energy $\Omega(\mathbf{R}, \mathbf{V}),{ }^{31}$

$$
E_{\text {elec }}(\mathbf{R}, \mathbf{V})=E_{\mathrm{KS}}(\mathbf{R}, \mathbf{V})+\Omega(\mathbf{R}, \mathbf{V}),
$$

where $E_{\mathrm{KS}}(\mathbf{R}, \mathbf{V})$ is calculated using the so-called solvated Fock operator:

$$
\hat{F}^{\text {solv }}=\hat{F}^{\mathrm{KS}}+\mathbf{V}^{T} \hat{\mathbf{Q}}
$$

Here $\hat{F}^{\mathrm{KS}}$ is the usual gas-phase Kohn-Sham Fock operator, and $\hat{\mathbf{Q}}$ is charge generation operator. Note that $E_{\mathrm{KS}}(\mathbf{R}, \mathbf{V})$ is the energy of the triplet state with two unpaired alpha electrons. The excitation energy $\Omega$ and transition amplitude $\mathbf{X}$ are determined by solving the collinear SFDFT equation ${ }^{31}$

$$
\mathbf{A X}=\Omega(\mathbf{V}) \mathbf{X}
$$

Here, no modification is applied to the coupling matrix $\mathbf{A}$ because the ESP $\mathbf{V}$ is thought of as an external potential. The excitation energy $\Omega$, however, has dependence on $\mathbf{V}$ through molecular orbital coefficients and orbital energies.

The equilibrium free energy for the target state is determined by minimizing the nonequilibrium free energy of Eq. (1) with respect to ESP V,

$$
\begin{aligned}
\frac{\partial G_{\text {noneq }}}{\partial \mathbf{V}} & =\frac{\partial E_{\text {elec }}}{\partial \mathbf{V}}-\mathbf{Q}_{0}+\frac{1}{\beta} \mathbf{C}_{0}^{-1}\left(\mathbf{V}-\mathbf{V}_{0}\right) \\
& \equiv \tilde{\mathbf{Q}}-\mathbf{Q}_{0}+\frac{1}{\beta} \mathbf{C}_{0}^{-1}\left(\mathbf{V}-\mathbf{V}_{0}\right) .
\end{aligned}
$$

$\tilde{\mathbf{Q}}$ is the derivative of $E_{\text {elec }}$ in Eq. (3) with respect to $\mathbf{V}$ and is given by $\tilde{\mathbf{Q}}=\operatorname{Tr}[(\mathbf{D}+\mathbf{P}) \mathbf{Q}]$, where $\mathbf{D}$ and $\mathbf{P}$ are the 
reference-state density matrix and excited-state relaxed difference density matrix, respectively. By setting $\partial G_{\text {noneq }} / \partial \mathbf{V}=0$, Eq. (6) leads to

$$
\mathbf{V}=\mathbf{V}_{0}-\beta \mathbf{C}_{0}\left(\tilde{\mathbf{Q}}-\mathbf{Q}_{0}\right) .
$$

Because the ESP depends on the site charge $\tilde{\mathbf{Q}}$, Eqs. (3) and (7) are solved to be self-consistent. The resultant equilibrium free energy is

$$
\begin{aligned}
G_{\text {eq }}(\mathbf{R})= & G_{\text {noneq }}\left(\mathbf{R}, \mathbf{V}_{\text {eq }}\right) \\
= & E_{\text {elec }}\left(\mathbf{R}, \mathbf{V}_{\text {eq }}\right)-\mathbf{V}_{\text {eq }}^{T} \mathbf{Q}_{0}+\Delta \mu_{0} \\
& +\frac{1}{2 \beta}\left(\mathbf{V}_{\text {eq }}-\mathbf{V}_{0}\right)^{T} \mathbf{C}_{0}^{-1}\left(\mathbf{V}_{\text {eq }}-\mathbf{V}_{0}\right) .
\end{aligned}
$$

The analytic free-energy gradient of Eqs. (1) and (8) is computed as in Ref. 22.

The free-energy crossing points are determined on the basis of nonequilibrium free energy [Eq. (1)] for relevant electronic states by modifying the branching plane (BP) update method $^{51}$ discussed in Sec. II B.

\section{B. Branching-space updating method}

The BP update method ${ }^{51}$ is adopted here to find the freeenergy crossing points in solution. This approach employs only the energy gradient vectors and does not require the nonadiabatic coupling vector that is computationally demanding for correlated electronic structure methods. Recently, Zhang and Herbert ${ }^{48}$ have implemented the analytic nonadiabatic coupling vector and optimized MECP. Although the original BP method is designed to locate the MECP in the gas phase, the extension is straightforward. The free-energy gradient for states $I$ and $J$ is obtained by taking the derivative with respect to the solvation coordinate $\mathbf{V}$ as well as the solute nuclear coordinate $\mathbf{R}$ :

$$
\mathbf{x}_{I}=\left(\begin{array}{l}
\partial G_{\text {noneq }}^{I} / \partial \mathbf{R} \\
\partial G_{\text {noneq }}^{I} / \partial \mathbf{V}
\end{array}\right), \quad \mathbf{x}_{J}=\left(\begin{array}{l}
\partial G_{\text {noneq }}^{J} / \partial \mathbf{R} \\
\partial G_{\text {noneq }}^{J} / \partial \mathbf{V}
\end{array}\right) .
$$

The dimension of these vectors is equal to four times the number of atoms.

The CI seam is characterized by two vectors: the difference gradient vector,

$$
\mathbf{f}_{I J} \equiv \nabla_{\xi}\left(G_{\text {noneq }}^{I}-G_{\text {noneq }}^{J}\right)=\mathbf{x}_{I}-\mathbf{x}_{J},
$$

and the derivative coupling vector,

$$
\mathbf{g}_{I J} \equiv\left\langle\Psi_{I} \mid \nabla_{\xi} \Psi_{I}\right\rangle
$$

Note that the subscript $\xi$ denotes the nuclear coordinate or the solvation coordinate.

The gradient employed in the MECP search consists of the sum of two terms: the gradient of squared free energy difference $\left(G_{\text {noneq }}^{I}-G_{\text {noneq }}^{J}\right)^{2}$ and the projected gradient of upper state $J$,

$$
2\left(G_{\text {noneq }}^{I}-G_{\text {noneq }}^{J}\right) \mathbf{f}_{I J}+\mathcal{P} \nabla_{\xi} G_{\text {noneq }}^{J} .
$$

The projection operator $\mathcal{P}$ is

$$
\mathcal{P}=1-\hat{\mathbf{f}}_{I J}\left(\hat{\mathbf{f}}_{I J}\right)^{T}-\hat{\mathbf{g}}_{I J}^{\perp}\left(\hat{\mathbf{g}}_{I J}^{\perp}\right)^{T},
$$

where

$$
\mathbf{g}_{I J}^{\perp}=\left(1-\hat{\mathbf{f}}_{I J} \hat{\mathbf{f}}_{I J}^{T}\right) \mathbf{g}_{I J} .
$$

The hat symbol means the unit vector. The BP update method uses the fact that the optimization requires the transformed $\mathbf{g}_{I J}^{\perp}$, not $\mathbf{g}_{I J}$ itself. The current vector $\mathbf{g}_{I J}^{\perp}$ is constructed as the linear combination of three vectors, the current and previous $\mathbf{f}_{I J}$ and the previous $\mathbf{g}_{I J}^{\perp}$, so as to satisfy the relation $\mathbf{f}_{I J}^{T} \mathbf{g}_{I J}^{\perp}=0$ at the current step. At the initial geometry, the average gradient $\left(\mathbf{x}_{I}+\mathbf{x}_{J}\right) / 2$ is used as an input.

\section{Computational details}

The LRFE-SFDFT method is applied to study the MECP of stilbene in an acetonitrile solution and those of thymine in water. The developed codes were interfaced with the program package GAMESS. ${ }^{52,53}$ The collinear SFDFT with the BHHLYP functional (50\% Hartree-Fock plus 50\% Becke exchange $^{54}$ with Lee-Yang-Parr correlation ${ }^{55}$ ) was used. Recently, Zhou et al. ${ }^{46}$ performed the SFDFT single-point energy calculations for model PSB with the meta-generalized gradient approximation functional, M05-2X, and observed the improvement of PES profile near the CI point. The functional dependence of CI geometries will be discussed in a future study. The basis set employed was cc-pVDZ. ${ }^{56}$ The reference triplet state was described using the restricted-open formulation.

The site-site RISM equation was solved with the hypernetted-chain closure relation. For acetonitrile solvent, threesite model by Jorgensen and Briggs ${ }^{57}$ was adopted, and the density was set to be $0.777 \mathrm{~g} / \mathrm{cm}^{3}$. For water solvent, simple point charge (SPC) model ${ }^{58}$ was used, and the density of $1.0 \mathrm{~g} / \mathrm{cm}^{3}$ was chosen. In all calculations, the temperature was set to be $298.15 \mathrm{~K}$. The solute partial charges were determined using the restrained ESP (RESP) fitting procedure, ${ }^{59}$ and the solute Lennard-Jones (LJ) parameters were taken from the AMBER force field. ${ }^{60}$ The standard combination rule was applied to compute the site-site LJ interaction potential.

\section{FREE ENERGY CROSSING POINTS IN SOLUTION}

Before the discussion of the calculated results for stilbene and thymine, a few remarks are presented. First, the solvation coordinate at the MECP is determined according to the nonequilibrium free energy, Eq. (1), and the resultant solvation coordinate does not necessarily reflect real solvation dynamics. The solute-solvent system is equilibrated in the ground state and has some memory after photoexcitation. At the excited state, the solute molecule finds a crossing seam on the way to a new equilibrium state. The nonequilibrium free energy considered in this work is not related to such nonequilibrium solvation dynamics. Second, the nonadiabatic transition does not have to occur at the MECP. It is likely that the nonadiabatic transition occurs efficiently once the energy gap is sufficiently small at some point that is higher in energy than the MECP. However, the energy difference between the excited-state minimum and MECP is a critical factor of photodynamics. On the assumption that the equilibra- 
tion is achieved at the excited-state minimum, one can predict that the relaxation via the MECP is slow for the endothermic reaction. The other points in the seam are much higher in energy than the MECP. Under strongly nonequilibrium condition, however, the free-energy difference may not directly explain the dynamics. The present calculations show that the excited-state minimum and crossing points are far below the Franck-Condon (FC) point, and the reaction does not always proceed through the lowest MECP. Rather, the MECP with higher energy may come into play when this point is accessible from the FC region.

\section{A. Stilbene in an acetonitrile solution}

The stilbene is the substituted ethylene and is a prototype molecule that undergoes the isomerization around the central carbon-carbon double bond. Recently, several theoretical calculations ${ }^{61-65}$ have examined the photoisomerization of stilbene. Spectroscopic studies ${ }^{66-68}$ detect the twisted minimum (so-called phantom state) and estimate the lifetime of $0.4(0.3)$ ps in an acetonitrile ( $n$-hexane) solution. The recent SFDFT calculation ${ }^{61}$ supports the existence of phantom state. That study determines twisted minimum and twistedpyramidalized MECP geometries in the gas phase and predicts the energy difference is $0.11 \mathrm{eV}$. The covalent and ionic states cross along the torsion and pyramidalization coordinates. Thus, locating the phantom state in solution depends critically on how much the polar solvent stabilizes the ionic state compared with the covalent state.

Figure 2 shows the optimized geometries at the twisted minimum, $\mathrm{S}_{1}$ (twisted), and MECP, and Table I collects the geometric parameters. Appreciable solvent effects are observed for both geometries. The pyramidalization angle of $\omega\left(\mathrm{C}_{8} \mathrm{C}_{1} \mathrm{C}_{2} \mathrm{H}_{15}\right)$ decreases by $7.9^{\circ}$ and $10.8^{\circ}$ for $\mathrm{S}_{1}$ (twisted) and MECP. The twisting around the ethylenic bond, $\tau\left(\mathrm{C}_{1} \mathrm{C}_{8}\right)$, increases from $80.3^{\circ}$ to $87.1^{\circ}$, and the molecule remains almost perpendicular in the solution-phase reaction from $\mathrm{S}_{1}$ (twisted) to MECP. The bending angle $\angle \mathrm{C}_{2} \mathrm{C}_{1} \mathrm{C}_{8}$ increases


FIG. 2. Optimized geometries for stilbene at (a) twisted minimum and (b) MECP. Geometries are superimposed: gas (red) and acetonitrile (blue).
TABLE I. Selected geometric parameters of stilbene. ${ }^{\text {a }}$ Bond lengths are in angstrom and angles are in degrees. Atom numbering is given in Fig. 1.

\begin{tabular}{lcc}
\hline \hline & $\mathrm{S}_{1}$ (twisted) & MECP \\
\hline$r\left(\mathrm{C}_{1} \mathrm{C}_{8}\right)$ & $1.429(1.418)$ & $1.426(1.418)$ \\
$r\left(\mathrm{C}_{1} \mathrm{C}_{2}\right)$ & $1.412(1.433)$ & $1.448(1.496)$ \\
$r\left(\mathrm{C}_{8} \mathrm{C}_{9}\right)$ & $1.396(1.409)$ & $1.394(1.423)$ \\
$\left\langle\mathrm{C}_{2} \mathrm{C}_{1} \mathrm{C}_{8}\right.$ & $119.9(116.9)$ & $112.3(96.7)$ \\
$\left\langle\mathrm{C}_{8} \mathrm{C}_{1} \mathrm{H}_{15}\right.$ & $109.9(108.1)$ & $103.3(107.0)$ \\
$\tau\left(\mathrm{C}_{1} \mathrm{C}_{8}\right)^{\mathrm{b}, \mathrm{c}}$ & $88.8(87.5)$ & $87.1(80.3)$ \\
$\tau\left(\mathrm{C}_{1} \mathrm{C}_{2}\right)^{\mathrm{b}}$ & $1.5(2.2)$ & $-0.8(27.2)$ \\
$\tau\left(\mathrm{C}_{8} \mathrm{C}_{9}\right)^{\mathrm{b}}$ & $0.5(0.5)$ & $-0.3(-3.5)$ \\
$\omega\left(\mathrm{C}_{8} \mathrm{C}_{1} \mathrm{C}_{2} \mathrm{H}_{15}\right)^{\mathrm{d}}$ & $38.8(46.7)$ & $58.7(69.5)$ \\
\hline \hline
\end{tabular}

${ }^{a}$ Values in parentheses are optimized in the gas phase.

${ }^{\mathrm{b}} \tau(i j)$ : average of four dihedral angles around bond $i-j$.

${ }^{\mathrm{c}}$ Value of $0^{\circ}\left(180^{\circ}\right)$ corresponds to the planar cis (trans) isomer.

${ }^{\mathrm{d}} \omega(i j k l)$ : out-of-plane angle from bond $i-j$ to plane $j-k-l$.

by $15.6^{\circ}$, and this is due to the decrease of $p$ character at the $\mathrm{C}_{1}$ atom as the pyramidalization is suppressed in solution. The rotation of the phenyl group, $\tau\left(C_{1} C_{2}\right)$, diminishes in transferring from the gas to solution phase, and the coplanar configuration in solution enhances the $\pi$-conjugation. In the gas phase, only the pyramidalization motion stabilizes the ionic state, because this mode minimizes the electric repulsion between the $p$ and bonding orbitals of the carbon atom. In acetonitrile solvent, however, strong solute-solvent interaction, which can also stabilize the ionic state, reduces the energy cost caused by large intramolecular distortion.

Figure 3 shows the solvation coordinate at the $\mathrm{S}_{0}$ (trans), $\mathrm{S}_{1}$ (trans), $\mathrm{S}_{1}$ (twisted), and MECP geometries. The solvation coordinate (ESP) is small at all atomic sites for the planar nonpolar conformations, $\mathrm{S}_{0}$ (trans) and $\mathrm{S}_{1}$ (trans). In contrast, large positive/negative values are observed for both $S_{1}$ (twisted) and MECP geometries. The ESP values are consistent with the solute electronic structure and indicate the dipolar solvation. Since the $\pi \pi^{*}$ state is ionic, the $\mathrm{C}_{2}-\mathrm{C}_{7}\left(\mathrm{C}_{9}-\mathrm{C}_{14}\right)$ atoms on the right (left) phenyl ring (see Fig. 2) have negative (positive) charges. Interestingly, the difference between the two solvation coordinates is negligible. The free energy is minimally dependent on the solvation coordinate in the reaction from $S_{1}$ (twisted) to MECP if the solvent equilibration is fast enough at $S_{1}$ (twisted).



FIG. 3. Solvation coordinates of stilbene at $S_{0}$ (trans) (red, solid), $S_{1}$ (trans) (green, dashed), $\mathrm{S}_{1}$ (twisted) (blue, dotted), and twisted-pyramidalized MECP (magenta, dashed-dotted). 
TABLE II. Relative energies (in eV) at the equilibrium and energy crossing points of stilbene. ${ }^{\mathrm{a}}$

\begin{tabular}{lccccc}
\hline \hline & \multicolumn{2}{c}{ Gas } & & \multicolumn{2}{c}{ Acetonitrile } \\
\cline { 2 - 3 } \cline { 6 - 7 } Geometry & $\mathrm{S}_{0}$ & $\mathrm{~S}_{1}$ & & $\mathrm{~S}_{0}$ & $\mathrm{~S}_{1}$ \\
\hline $\mathrm{S}_{0}$ (trans) & $0.00(0.01)$ & $4.42(0.07)$ & & $0.00(0.01)$ & $4.42(0.07)$ \\
$\mathrm{S}_{1}$ (trans) & $0.36(0.02)$ & $4.01(0.05)$ & & $0.36(0.02)$ & $4.01(0.05)$ \\
$\mathrm{S}_{1}$ (twisted) & $3.02(0.87)$ & $4.03(0.07)$ & & $3.20(0.88)$ & $3.84(0.06)$ \\
MECP & $4.21(0.49)$ & $4.21(0.45)$ & & $3.92(0.64)$ & $3.92(0.36)$ \\
\hline \hline
\end{tabular}

${ }^{a}$ Values in parentheses are spin expectation values $\left\langle S^{2}\right\rangle$.

In order to examine how the solvent assists the MECP in solution, the reorganization energy $\lambda^{I}$ is computed at the MECP geometry:

$$
\lambda^{I}=G_{\text {noneq }}^{I}\left(\mathbf{R}_{\mathrm{MECP}}, \mathbf{V}_{\mathrm{MECP}}\right)-G_{\text {noneq }}^{I}\left(\mathbf{R}_{\mathrm{MECP}}, \mathbf{V}_{\text {eq }}^{I}\right) .
$$

The lower covalent state has the energy of $9.7 \mathrm{kcal} / \mathrm{mol}$. For the upper ionic state, however, the minimization of $G_{\text {noneq }}^{I}$ with respect to $\mathbf{V}$ leads to the oscillation around the MECP. The result is consistent with the fact that $\mathbf{V}_{\text {MECP }}$ also shows the dipolar solvation. Therefore, the dominant role of the nonequilibrium solvation is to destabilize the covalent state in achieving the degeneracy.

Table II shows the relative energies in the gas and solution phases. There is negligible solvatochromic shift for absorption and emission energies. Contrastively, the $\pi \pi^{*}$-state energy at the $S_{1}$ (twisted) geometry decreases by $0.19 \mathrm{eV}$ due to the strong ionic character: the dipole moment of 12.34 (8.71) D in solution (vacuum). At the same time, the ground-state energy increases by $0.18 \mathrm{eV}$ because the dipolar solvation destabilizes the covalent state. For the MECP, the energy decreases by $\sim 0.3 \mathrm{eV}$. Although the optimal solvation coordinate is similar with that at $\mathrm{S}_{1}$ (twisted) and reflects the dipolar solvation, the polar solvent helps reducing the intramolecular distortion (e.g., pyramidalization motion) that disfavors the radical ("dot") on the carbon. The energy difference between the $S_{1}$ (twisted) and MECP is $0.08(0.18)$ $\mathrm{eV}$ in acetonitrile (vacuum), indicating that the reaction proceeds faster in solution. Note that the discussion assumes that the molecule relaxes to the equilibrium $S_{1}$ (twisted) minimum and that the free-energy difference explains the reaction rate in solution. The equilibrium $\mathrm{S}_{1}$ (twisted) minimum considered in this work does not necessary represents the phantom state found by the experiments. It is not obvious that true equilibrium solvation is achieved at the excited-state minimum in solution. Furthermore, the free-energy difference may not directly affect the photodynamics under the strong nonequilibrium condition. The solvent reorganization from nonpolar to dipolar solvation plays a decisive role in controlling the reaction rate.

To examine how the solvent participates in the reaction, the two-dimensional free-energy map connecting the $\mathrm{S}_{1}$ (twisted) and MECP points is constructed. Note that the discussion based on the free-energy profile may be inappropriate under strong nonequilibrium condition (see above). The solute geometries are generated using the linear synchronous transit (LST) method, ${ }^{70}$ while the solvation coordinate is simply interpolated. The energy profile is shown in Fig. 4.

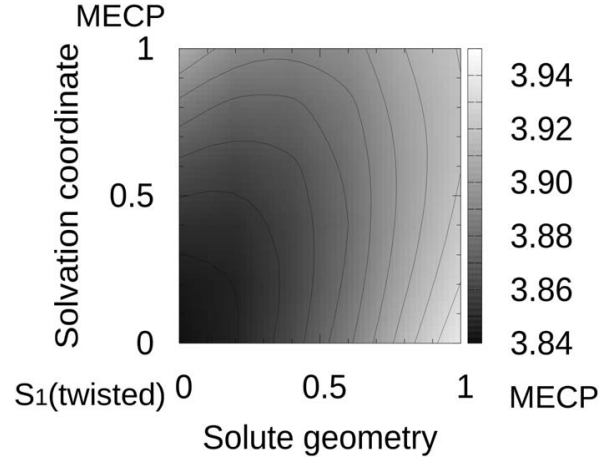

FIG. 4. Two-dimensional free energy profile of stilbene along the solute and solvation coordinates. The origin corresponds to the equilibrium $\mathrm{S}_{1}$ (twisted) and $(1.0,1.0)$ to MECP. Unit: eV.

The free energy increases monotonically from $\mathrm{S}_{1}$ (twisted) (3.84 eV) to MECP (3.92 eV), and there is no barrier that separates these two geometries. Around the origin (FC region), the energy is relatively flat along the solvation coordinate and this is consistent with the similarity of the ESP (see Fig. 3). As the weight of MECP increases the geometric changes become a dominant reaction coordinate; the contour is parallel with the solvation coordinate axis.

The present calculations, however, cannot be directly compared with the photoisomerization dynamics measured in the experiments. Kovalenko and Dobryakov ${ }^{69}$ suggest that a simple rate theory is not enough to describe the kinetics in solution and that the reaction needs the activation by solvent collisions. In this point, the nonadiabatic simulation could give the insights into stilbene dynamics in solution.

\section{B. Thymine in water}

Thymine is one of the DNA bases and the radiationless transition play an essential role in protecting the genetic code against harmful ultraviolet light. The photostability of thymine has been the subject of both theoretical and experimental studies. ${ }^{71-87}$ Several computational studies ${ }^{71-74}$ located the MECP geometries by various electronic structure methods and proposed the reaction path connecting the FC and MECP. The nonadiabatic simulation has also been performed to examine the relaxation pathways of excited-state thymine. $^{75-78}$

Recently, Nakayama et $a l .{ }^{88}$ considered the three MECP structures between the $\mathrm{S}_{0}$ and $\pi \pi^{*}$ states: $\operatorname{MECP}\left(\mathrm{C}_{5}\right)$, $\operatorname{MECP}\left(\mathrm{C}_{6}\right)$, and $\operatorname{MECP}\left(\mathrm{C}_{4}\right)$. The $\operatorname{MECP}\left(\mathrm{C}_{5}\right)$ and $\operatorname{MECP}\left(\mathrm{C}_{6}\right)$ structures are puckered at the $\mathrm{C}_{5}$ and $\mathrm{C}_{6}$ position (for atom numbering, see Fig. 1), respectively, and are similar to the CI of ethylene. In contrast, the $\operatorname{MECP}\left(\mathrm{C}_{4}\right)$ is puckered at $\mathrm{C}_{4}$ position, and the carbonyl oxygen bends out of the molecular plane. These authors have performed the QM/MM MD simulation of aqueous thymine by using the second-order perturbation theory with the complete active space SCF reference (CASPT2) and found an additional relaxation path in the water. Three of ten trajectories go to the $\operatorname{MECP}\left(\mathrm{C}_{4}\right)$ in water while the transition seems to occur exclusively via $\operatorname{MECP}\left(\mathrm{C}_{5}\right)$ in a vacuum. The branch indicates that the relaxation path to the $\operatorname{MECP}\left(\mathrm{C}_{4}\right)$ is open in water due to the favorable 
solute-solvent interaction. The present work elucidates the role of $\operatorname{MECP}\left(\mathrm{C}_{4}\right)$ in water on the basis of free-energy surface crossing. The discussion relying only on the FC and MECP may be insufficient because the deactivation mechanism depends on the global character of the (free) energy surface as well as the energies of these points. The excited-state MD simulation, which begins at the FC point, can take accounts of the nonequilibrium solvation effect naturally, while the solvation coordinate in this work does not necessarily reflect the real solvent relaxation.

Figure 5 shows the MECP geometries optimized in the gas and aqueous solution phases. The bond lengths obtained by the CASPT2 method ${ }^{88}$ are also included for comparison. The geometries obtained by the SFDFT with BHHLYP functional reproduce those by the CASPT2 qualitatively. The difference is $\sim 0.05 \AA$ for $\operatorname{MECP}\left(\mathrm{C}_{6}\right)$ and $\operatorname{MECP}\left(\mathrm{C}_{4}\right)$ and $\sim 0.07 \AA$ for $\operatorname{MECP}\left(\mathrm{C}_{5}\right)$. The $\operatorname{MECP}\left(\mathrm{C}_{5}\right)$ and $\operatorname{MECP}\left(\mathrm{C}_{6}\right)$ are



FIG. 5. Optimized geometries of thymine at (a) $\operatorname{MECP}\left(\mathrm{C}_{5}\right)$, (b) $\operatorname{MECP}\left(\mathrm{C}_{6}\right)$, and (c) $\operatorname{MECP}\left(\mathrm{C}_{4}\right)$ in vacuum (red) and water (blue). Bond lengths are given in angstrom: gas (top), CASPT2 values taken from Ref. 88 (middle, italic), and water (bottom). marginally dependent on the presence of polar solvent. The geometric changes are expected since these structures are associated with the MECP of ethylene. For the $\operatorname{MECP}\left(\mathrm{C}_{5}\right)$, the pyramidalization angle $\omega\left(\mathrm{C}_{9} \mathrm{C}_{5} \mathrm{C}_{4} \mathrm{C}_{6}\right)$ is suppressed in water $\left(47.3^{\circ} \rightarrow 40.5^{\circ}\right)$. For the $\operatorname{MECP}\left(\mathrm{C}_{6}\right)$, the change in pyramidalization angle $\omega\left(\mathrm{H}_{12} \mathrm{C}_{6} \mathrm{C}_{1} \mathrm{C}_{5}\right)$ is small $\left(80.7^{\circ} \rightarrow 78.4^{\circ}\right)$. The angle $\angle \mathrm{H}_{12} \mathrm{C}_{6} \mathrm{C}_{5}$ increases $\left(83.3^{\circ} \rightarrow 91.3^{\circ}\right)$ and the $\mathrm{C}_{6} \mathrm{H}_{12}$ bond shrinks by $0.03 \AA$, and these two parameters indicate the degree of hydrogen migration is smaller in the water. On the other hand, large geometric changes are observed for the $\operatorname{MECP}\left(\mathrm{C}_{4}\right)$. The torsion angles in the ring differ significantly. Moreover, the geometric changes around the carbonyl group are remarkable: the $\mathrm{C}_{3} \mathrm{C}_{4}$ and $\mathrm{C}_{4} \mathrm{C}_{5}$ bonds stretch by 0.04 and $0.09 \AA$, respectively, and the bending angle $\angle \mathrm{O}_{8} \mathrm{C}_{4} \mathrm{C}_{5}$ increases $\left(83.4^{\circ} \rightarrow 101.6^{\circ}\right)$. In other words, the solvent pulls the carbonyl group out of the remaining part in water.

The CI geometries depend critically on the choice of functional for the SF-TDDFT method, and a benchmark study on this topic is now in progress. The preliminary results of ethylene show that decreasing the Hartree-Fock exchange suppresses the out-of-plane motion. Here, the three CI geometries are optimized using the B3LYP functional, although this is not the primary purpose of this work. The B3LYP provides the energy of $4.48,4.75$, and $5.73 \mathrm{eV}$ for the $\operatorname{MECP}\left(\mathrm{C}_{5}\right)$, $\operatorname{MECP}\left(\mathrm{C}_{6}\right)$, and $\operatorname{MECP}\left(\mathrm{C}_{4}\right)$, respectively. This result gives the same energetic order observed for the BHHLYP. As for the CI geometry, the pyramidalization angle $\omega\left(\mathrm{H}_{12} \mathrm{C}_{6} \mathrm{~N}_{1} \mathrm{C}_{5}\right)$ of $\operatorname{MECP}\left(\mathrm{C}_{6}\right)$ decreases from $80.6^{\circ}$ (BHHLYP) to $49.9^{\circ}$ (B3LYP) as in the case of ethylene. In contrast, the outof-plane motion of the carbonyl group does not change for $\operatorname{MECP}\left(\mathrm{C}_{4}\right)$ : the pyramidalization angle $\omega\left(\mathrm{O}_{8} \mathrm{C}_{4} \mathrm{~N}_{3} \mathrm{C}_{5}\right)$ is $55.2^{\circ}\left(52.4^{\circ}\right)$ for the B3LYP (BHHLYP).

Figure 6 shows the solvation coordinates that realizes the crossing between the nonequilibrium free energy surfaces. The deviation from the ground-state equilibrium solvation is small for the $\operatorname{MECP}\left(\mathrm{C}_{6}\right)$ and $\operatorname{MECP}\left(\mathrm{C}_{5}\right)$. The ESP at the $\mathrm{C}_{6}$ atom is positive for the $\operatorname{MECP}\left(\mathrm{C}_{6}\right)$ because the pyramidalization induces the negative charge on that atom, and the same is true for the $\mathrm{C}_{5}$ atom of $\operatorname{MECP}\left(\mathrm{C}_{5}\right)$. In contrast, drastic changes are observed for the $\operatorname{MECP}\left(\mathrm{C}_{4}\right)$. In particular, the carbonyl oxygen has large positive ESP, indicating that the $\pi \pi^{*}$ excitation enhances the hydrogen bond between the oxygen and water hydrogen atoms.

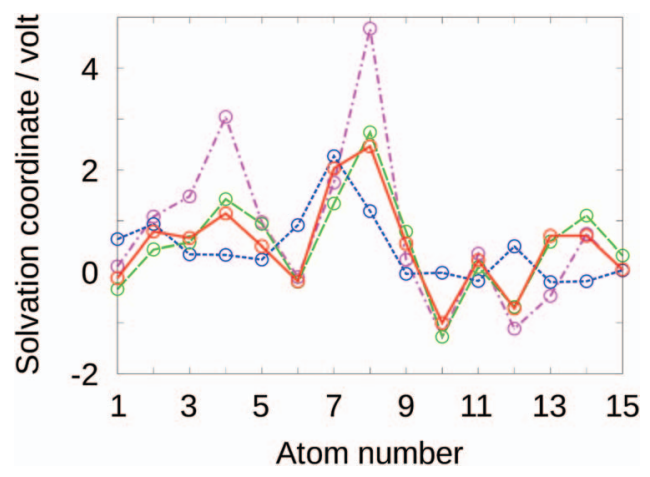

FIG. 6. Solvation coordinates of thymine at $\mathrm{S}_{0}$ minimum (red, solid), $\operatorname{MECP}\left(\mathrm{C}_{5}\right)$ (green, dashed), $\operatorname{MECP}\left(\mathrm{C}_{6}\right)$ (blue, dotted), and $\operatorname{MECP}\left(\mathrm{C}_{4}\right)$ (magenta, dashed-dotted). 
TABLE III. Relative energies (in eV) at the equilibrium and energy crossing points of thymine. ${ }^{\mathrm{a}}$

\begin{tabular}{lccccc}
\hline \hline & \multicolumn{2}{c}{ Gas } & & \multicolumn{2}{c}{ Water } \\
\cline { 2 - 3 } \cline { 5 - 6 } Geometry & $\mathrm{S}_{0}$ & $\mathrm{~S}_{1}$ & & $\mathrm{~S}_{0}$ & $\mathrm{~S}_{1}$ \\
\hline $\mathrm{S}_{0}$ & $0.00(0.01)$ & $5.92^{\mathrm{b}}(0.12)$ & & $0.00(0.01)$ & $5.79^{\mathrm{b}}(0.12)$ \\
$\operatorname{MECP}\left(\mathrm{C}_{5}\right)$ & $4.54(0.59)$ & $4.54^{\mathrm{c}}(0.27)$ & & $4.48(0.24)$ & $4.48(0.23)$ \\
$\operatorname{MECP}\left(\mathrm{C}_{6}\right)$ & $5.52(0.31)$ & $5.52^{\mathrm{c}}(0.53)$ & & $5.53(0.61)$ & $5.53(0.21)$ \\
$\operatorname{MECP}\left(\mathrm{C}_{4}\right)$ & $5.60(0.15)$ & $5.60^{\mathrm{c}}(0.32)$ & & $4.94(0.27)$ & $4.94(0.34)$ \\
\hline \hline
\end{tabular}

${ }^{a}$ Values in parentheses are spin expectation values $\left\langle S^{2}\right\rangle$.

${ }^{b}$ Experimental absorption energy is $4.95 \pm 0.08 \mathrm{eV}$ in vacuum (Ref. 85) and $4.68 \mathrm{eV}$ in water (Ref. 86). Theoretical values are summarized: $5.28 \mathrm{eV}$ by the resolution-ofidentity approximate second-order coupled cluster (RI-CC2) in Ref. 77, $5.69 \mathrm{eV}$ by the equation-of-motion coupled cluster with single and double excitations (EOM-CCSD) in Ref. 73 , and $4.83 \mathrm{eV}$ by the multi-state CASPT2 in Refs. 74 and 88.

${ }^{\mathrm{c}}$ Multi-state CASPT2 energies in Ref. 88 are 4.13/4.15, 4.79/4.86, and 4.80/4.94 eV for $\operatorname{MECP}\left(\mathrm{C}_{5}\right), \operatorname{MECP}\left(\mathrm{C}_{6}\right)$, and $\operatorname{MECP}\left(\mathrm{C}_{4}\right)$, respectively.

The nonequilibrium solvation is examined using the solvent reorganization energy [Eq. (15)]. At the $\operatorname{MECP}\left(\mathrm{C}_{5}\right)$ and $\operatorname{MECP}\left(\mathrm{C}_{6}\right)$, the covalent state has the energy of 2.9 and $5.3 \mathrm{kcal} / \mathrm{mol}$ while the equilibrium ionic state is not found as in the case of stilbene. The reorganization energy of the $\operatorname{MECP}\left(\mathrm{C}_{4}\right)$ is $\sim 0.0(28.8) \mathrm{kcal} / \mathrm{mol}$ for the open- (closed-) shell state. Note that the polarity of the open-shell state is larger than that of the closed-shell for the $\operatorname{MECP}\left(\mathrm{C}_{4}\right)$. For all three MECPs considered in this work, the nonequilibrium solvation raises the energy of weakly polar state to achieve the $\mathrm{CI}$ in solution.

Table III summarizes the energy computed at the SFBHHLYP/cc-pVDZ level. Compared to the CASPT2 calculation and experiments, the energy values are uniformly overestimated. However, the present calculations reproduce a general trend. The absorption energy is red-shifted by $0.13 \mathrm{eV}$, which is comparable to the TDDFT/polarizable continuum model approach: $0.09 \mathrm{eV}$. In the gas phase, the obtained energetic order is $\operatorname{MECP}\left(\mathrm{C}_{5}\right)<\operatorname{MECP}\left(\mathrm{C}_{6}\right)$ $<\operatorname{MECP}\left(\mathrm{C}_{4}\right)$, and the latter two are $\sim 1.0 \mathrm{eV}$ above the $\operatorname{MECP}\left(\mathrm{C}_{5}\right)$. The $\operatorname{MECP}\left(\mathrm{C}_{6}\right)$ and $\operatorname{MECP}\left(\mathrm{C}_{5}\right)$ geometries have the similarity with the MECP of ethylene, and the functional groups attached to ethylenic carbon atoms determine the relative stability. For the $\operatorname{MECP}\left(\mathrm{C}_{6}\right)$, the $\mathrm{C}_{6}$ atom is pyramidalized, and the bond is ionic: $\mathrm{C}_{5}{ }^{+}-\mathrm{C}_{6}{ }^{-}$. The positive $\mathrm{C}_{5}$ (negative $\mathrm{C}_{6}$ ) atom is next to the electron-donating $\mathrm{N}_{1}$ (electron-withdrawing carbonyl $\mathrm{C}_{4}$ ) atom, and the $\pi \pi^{*}$ state is efficiently stabilized by these functional groups. Contrastively, the $\pi \pi^{*}$ state of $\operatorname{MECP}\left(\mathrm{C}_{5}\right)$ is polarized in the opposite direction, $\mathrm{C}_{5}{ }^{-}-\mathrm{C}_{6}{ }^{+}$, for the $\pi \pi^{*}$ state, and the neighboring groups destabilized such electronic distribution. In addition to the substituent effects, the solute-solvent interaction also contributes to the stability of MECP structures. The water solvent stabilizes significantly the $\operatorname{MECP}\left(\mathrm{C}_{4}\right)$ $(\sim 0.7 \mathrm{eV})$, which is higher in energy only by $0.46 \mathrm{eV}$ than the $\operatorname{MECP}\left(\mathrm{C}_{5}\right)$. In contrast, no substantial changes are observed for the $\operatorname{MECP}\left(\mathrm{C}_{5}\right)$ and $\operatorname{MECP}\left(\mathrm{C}_{6}\right)$. As shown in Fig. 6, the hydrogen bond formation plays a critical role in stabilizing the $\operatorname{MECP}\left(\mathrm{C}_{4}\right)$.

Figure 7 shows the two-dimensional maps from FC to MECP. Note that the barrier found along the path does not (a)



(b)

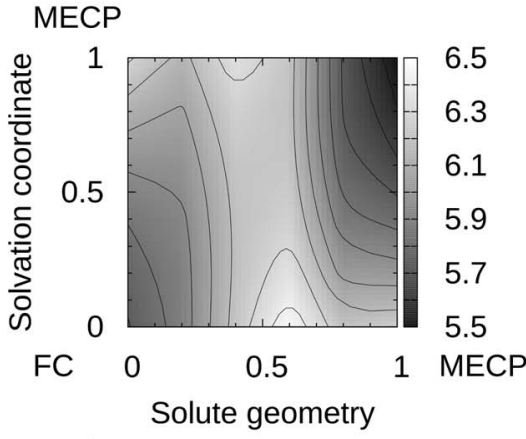

(c)

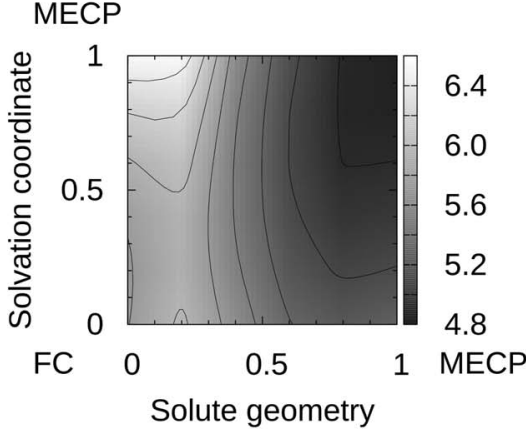

FIG. 7. Two-dimensional free energy profiles of thymine along the solute and solvation coordinates. From FC point to (a) $\operatorname{MECP}\left(\mathrm{C}_{5}\right)$, (b) $\operatorname{MECP}\left(\mathrm{C}_{6}\right)$, and (c) $\operatorname{MECP}\left(\mathrm{C}_{4}\right)$. The origin corresponds to $\mathrm{FC}$ and $(1.0,1.0)$ to $\mathrm{MECP}$. Unit: eV.

represent a true transition state, because the energy calculation uses the interpolated coordinates. The barrier height is always higher than that along the minimum energy path. In addition, the free-energy profiles may not directly reflect the dynamics in solution under the strong nonequilibrium condition. The three MECPs are far below the FC point, and thus, the solute molecule has sufficient energy. Not the relative stability of these geometries but the accessibility from the FC controls the reaction path. Therefore, the discussion below should be taken with caution. For the $\operatorname{MECP}\left(\mathrm{C}_{5}\right)$, the free energy decreases monotonically from the $\mathrm{FC}(5.79 \mathrm{eV})$ to MECP (4.48 eV), and the reaction seems to proceed without any barrier. Notably, the free energy is flat along the solvation coordinate, and this is due to the small deviation of ESP (see Fig. 6). For the $\operatorname{MECP}\left(\mathrm{C}_{6}\right)$, there is a large barrier of $0.45 \mathrm{eV}$ between the FC (5.79 eV) and MECP (5.53) points. The same trend is observed for the gas-phase energy profile at the CASPT2 level. ${ }^{88}$ Thus, this MECP may play a minor role in the dynamics (however, see the discussion above). The $\operatorname{MECP}\left(\mathrm{C}_{4}\right)$ map gives an interesting picture; there is a small barrier of $0.18 \mathrm{eV}$ between the FC $(5.79 \mathrm{eV})$ and MECP $(4.94 \mathrm{eV})$ points. The molecule leads to the MECP sponta- 
neously after crossing the barrier. This trend is consistent with the recent $\mathrm{MD}$ simulation ${ }^{88}$ proposing the nonadiabatic transition via $\operatorname{MECP}\left(\mathrm{C}_{4}\right)$ as well as $\operatorname{MECP}\left(\mathrm{C}_{5}\right)$ in water.

\section{CONCLUDING REMARKS}

In the present paper, the CI point in solution is determined as the crossing between nonequilibrium free energy surfaces for relevant electronic states. The nonequilibrium free energy is described using the LRFE-SFDFT method, which defines the ESP acting on the solute atoms as the solvation coordinate. The modified BP update method locates successfully the CI points in solution. The proposed method is applied to study (1) the photoisomerization of stilbene in an acetonitrile solution and (2) the nonadiabatic processes of thymine in water. The solvent effects on the dynamics of these molecules are examined on the basis of the free-energy profiles although it is not evident that the free energy reflects the real dynamics in solution under the strong nonequilibrium condition. This point should be clarified in the future work. Remarkable solvent effects are observed for both molecular systems. For stilbene, polar acetonitrile solvent decreases the energy difference between the twisted minimum and twisted-pyramidalized CI by $0.10 \mathrm{eV}$, although strong ionic character for the $\pi \pi^{*}$ state stabilizes both geometries by 0.19 and $0.29 \mathrm{eV}$. For thymine in water, forming a hydrogen bond stabilizes the CI puckered at the carbonyl carbon atom, $\operatorname{MECP}\left(\mathrm{C}_{4}\right)$, which is higher in energy by $0.46 \mathrm{eV}$ than the lowest $\operatorname{MECP}\left(\mathrm{C}_{5}\right)$. Furthermore, the free-energy map shows a small barrier of $0.18 \mathrm{eV}$ from the FC to MECP. These results are consistent with the recent simulation showing the transition via $\operatorname{MECP}\left(\mathrm{C}_{4}\right)$ is possible in water, although a direct comparison should be taken with caution. Therefore, the present method is a promising way of characterizing the solution-phase CI points that play an essential role in photodynamics of molecules in solution.

\section{ACKNOWLEDGMENTS}

Financial support by the research fund from Kyoto University is gratefully acknowledged.

${ }^{1}$ D. R. Yarkony, Chem. Rev. 112, 481 (2012).

${ }^{2}$ S. Matsika and P. Krause, Annu. Rev. Phys. Chem. 62, 621 (2011).

${ }^{3}$ B. G. Levine and T. J. Martínez, Annu. Rev. Phys. Chem. 58, 613 (2007).

${ }^{4}$ F. Bernardi, M. Olivucci, and M. A. Robb, Chem. Soc. Rev. 25, 321 (1996).

${ }^{5}$ A. M. Virshup, C. Punwong, T. V. Pogorelov, B. A. Lindquist, C. Ko, and T. J. Martínez, J. Phys. Chem. B 113, 3280 (2009).

${ }^{6}$ C. E. Crespo-Hernández, B. Cohen, P. M. Hare, and B. Kohler, Chem. Rev. 104, 1977 (2004).

${ }^{7}$ C. T. Middleton, K. de La Harpe, C. Su, Y. K. Law, C. E. Crespo- Hernández, and B. Kohler, Annu. Rev. Phys. Chem. 60, 217 (2009).

${ }^{8}$ M. Squillacote, J.-S. Wang, and J.-W. Chen, J. Am. Chem. Soc. 126, 1940 (2004)

${ }^{9}$ X. F. Xu, A. Kahan, S. Zilberg, and Y. Haas, J. Phys. Chem. A 113, 9779 (2009).

${ }^{10}$ A. Kahan, A. Wand, S. Ruhman, S. Zilberg, and Y. Haas, J. Phys. Chem. A 115, 10854 (2011)

${ }^{11}$ A. Toniolo, G. Granucci, and T. J. Martínez, J. Phys. Chem. A 107, 3822 (2003)

${ }^{12}$ I. Burghardt, L. S. Cederbaum, and J. T. Hynes, Faraday Discuss. 127, 395 (2004).

${ }^{13}$ R. Spezia, I. Burghardt, and J. T. Hynes, Mol. Phys. 104, 903 (2006).

${ }^{14}$ I. Burghardt and J. T. Hynes, J. Phys. Chem. A 110, 11411 (2006).
${ }^{15}$ J. P. Malhado, R. Spezia, and J. T. Hynes, J. Phys. Chem. A 115, 3720 (2011).

${ }^{16}$ J. P. Malhado and J. T. Hynes, J. Chem. Phys. 137, 22 A543 (2012).

${ }^{17}$ A. M. Losa, M. E. Martín, I. F. Galván, and M. A. Aguilar, Chem. Phys. Lett. 443, 76 (2007).

${ }^{18}$ A. M. Losa, I. F. Galván, M. L. Sánchez, M. E. Martín, and M. A. Aguilar, J. Phys. Chem. B 112, 877 (2008).

${ }^{19}$ G. Cui and W. Yang, J. Chem. Phys. 134, 204115 (2011).

${ }^{20}$ S. Yamazaki and S. Kato, J. Chem. Phys. 123, 114510 (2005).

${ }^{21}$ S. Yamazaki and S. Kato, J. Am. Chem. Soc. 129, 2901 (2007).

${ }^{22}$ T. Mori, K. Nakano, and S. Kato, J. Chem. Phys. 133, 064107 (2010).

${ }^{23}$ M. Higashi, S. Hayashi, and S. Kato, J. Chem. Phys. 126, 144503 (2007).

${ }^{24}$ T. Yamamoto and S. Kato, J. Chem. Phys. 126, 224514 (2007).

${ }^{25}$ S. Aono, N. Minezawa, and S. Kato, Chem. Phys. Lett. 492, 193 (2010).

${ }^{26}$ R. M. Levy, M. Belhadj, and D. B. Kitchen, J. Chem. Phys. 95, 3627 (1991).

${ }^{27}$ K. Naka, A. Morita, and S. Kato, J. Chem. Phys. 110, 3484 (1999).

${ }^{28}$ S. Ten-no, F. Hirata, and S. Kato, J. Chem. Phys. 100, 7443 (1994).

${ }^{29}$ H. Sato, F. Hirata, and S. Kato, J. Chem. Phys. 105, 1546 (1996).

${ }^{30} \mathrm{H}$. Sato, in Molecular Theory of Solvation, edited by F. Hirata (Kluwer Academic, Dordrecht, 2003), pp. 61-99.

${ }^{31}$ Y. Shao, M. Head-Gordon, and A. I. Krylov, J. Chem. Phys. 118, 4807 (2003).

${ }^{32}$ F. Wang and T. Ziegler, J. Chem. Phys. 121, 12191 (2004).

${ }^{33}$ F. Wang and T. Ziegler, J. Chem. Phys. 122, 074109 (2005).

${ }^{34}$ Z. Rinkevicius, O. Vahtras, and H. Ågren, J. Chem. Phys. 133, 114104 (2010).

${ }^{35}$ Z. Li and W. Liu, J. Chem. Phys. 136, 024107 (2012).

${ }^{36}$ Y. A. Bernard, Y. Shao, and A. I. Krylov, J. Chem. Phys. 136, 204103 (2012).

${ }^{37}$ M. Isegawa and D. G. Truhlar, J. Chem. Phys. 138, 134111 (2013).

${ }^{38}$ E. Epifanovsky and A. I. Krylov, Mol. Phys. 105, 2515 (2007).

${ }^{39}$ S. Gozem, A. I. Krylov, and M. Olivucci, J. Chem. Theory Comput. 9, 284 (2013).

${ }^{40}$ S. Gozem, F. Melaccio, R. Lindh, A. I. Krylov, A. A. Granovsky, C. Angeli, and M. Olivucci, J. Chem. Theory Comput. 9, 4495 (2013).

${ }^{41}$ X. Xu, S. Gozem, M. Olivucci, and D. G. Truhlar, J. Phys. Chem. Lett. 4, 253 (2013)

${ }^{42}$ M. Huix-Rotllant, B. Natarajan, A. Ipatov, C. M. Wawire, T. Deutsch, and M. E. Casida, Phys. Chem. Chem. Phys. 12, 12811 (2010).

${ }^{43}$ N. Minezawa and M. S. Gordon, J. Phys. Chem. A 113, 12749 (2009).

${ }^{44}$ N. Minezawa and M. S. Gordon, J. Chem. Phys. 137, 034116 (2012).

${ }^{45}$ Y. Harabuchi, S. Maeda, T. Taketsugu, N. Minezawa, and K. Morokuma, J. Chem. Theory Comput. 9, 4116 (2013).

${ }^{46}$ P. Zhou, J. Liu, K. Han, and G. He, J. Comput. Chem. 35, 109 (2014).

${ }^{47}$ X. Zhang and J. M. Herbert, J. Phys. Chem. B 118, 7806 (2014).

${ }^{48}$ X. Zhang and J. M. Herbert, J. Chem. Phys. 141, 064104 (2014).

${ }^{49}$ S. Gozem, F. Melaccio, A. Valentini, M. Filatov, M. Huix-Rotllant, N. Ferré, L. M. Frutos, C. Angeli, A. I. Krylov, A. A. Granovsky, R. Lindh, and M. Olivucci, J. Chem. Theory Comput. 10, 3074 (2014).

${ }^{50}$ N. Minezawa, Chem. Phys. Lett. 608, 140 (2014).

${ }^{51}$ S. Maeda, K. Ohno, and K. Morokuma, J. Chem. Theory Comput. 6, 1538 (2010).

${ }^{52}$ M. W. Schmidt, K. K. Baldridge, J. A. Boatz, S. T. Elbert, M. S. Gordon, J. H. Jensen, S. Koseki, N. Matsunaga, K. A. Nguyen, S. J. Su, T. L. Windus, M. Dupuis, and J. A. Montgomery, Jr., J. Comput. Chem. 14, 1347 (1993).

${ }^{53}$ M. S. Gordon and M. W. Schmidt, in Theory and Applications of Computational Chemistry: The First Forty Years, edited by C. E. Dykstra, G. Frenking, K. S. Kim, and G. E. Scuseria (Elsevier, Amsterdam, 2005), Chap. 41, pp. $1167-1189$.

${ }^{54}$ A. D. Becke, Phys. Rev. A 38, 3098 (1988).

${ }^{55}$ C. Lee, W. Yang, and R. G. Parr, Phys. Rev. B 37, 785 (1988).

${ }^{56}$ T. H. Dunning, Jr., J. Chem. Phys. 90, 1007 (1989).

${ }^{57}$ W. L. Jorgensen and J. M. Briggs, Mol. Phys. 63, 547 (1988).

${ }^{58}$ H. J. C. Berendsen, J. P. M. Postma, M. F. van Gunsteren, and J. Hermans, in Intermolecular Forces, edited by B. Pullman (Reidel, Dordrecht, 1981), p. 331.

${ }^{59}$ C. I. Bayly, P. Cieplak, W. D. Cornell, and P. A. Kollman, J. Phys. Chem. 97, 10269 (1993).

${ }^{60}$ W. D. Cornell, P. Cieplak, C. I. Bayly, I. R. Gould, K. M. Merz, Jr., D. M. Ferguson, D. C. Spellmeyer, T. Fox, J. W. Caldwell, and P. A. Kollman, J. Am. Chem. Soc. 117, 5179 (1995).

${ }^{61}$ N. Minezawa and M. S. Gordon, J. Phys. Chem. A 115, 7901 (2011) and references herein. 
${ }^{62}$ J. Quenneville and T. J. Martínez, J. Phys. Chem. A 107, 829 (2003).

${ }^{63}$ R. Improta and F. Santoro, J. Phys. Chem. A 109, 10058 (2005).

${ }^{64}$ I. N. Ioffe and A. A. Granovsky, J. Chem. Theory Comput. 9, 4973 (2013).

${ }^{65} \mathrm{G}$. Tomasello, M. Garavelli, and G. Orlandi, Phys. Chem. Chem. Phys. 15, 19763 (2013).

${ }^{66}$ W. Fuß, C. Kosmidis, W. E. Schmid, and S. A. Trushin, Chem. Phys. Lett. 385, 423 (2004).

${ }^{67}$ S. A. Kovalenko, A. L. Dobryakov, I. Ioffe, and N. P. Ernsting, Chem. Phys. Lett. 493, 255 (2010).

${ }^{68}$ F. Berndt, A. L. Dobryakov, M. Quick, R. Mahrwald, N. P. Ernsting, D. Lenoir, and S. A. Kovalenko, Chem. Phys. Lett. 544, 39 (2012).

${ }^{69}$ S. A. Kovalenko and A. L. Dobryakov, Chem. Phys. Lett. 570, 56 (2013).

${ }^{70}$ T. A. Halgren and W. N. Lipscomb, Chem. Phys. Lett. 49, 225 (1977).

${ }^{71}$ S. Perun, A. L. Sobolewski, and W. Domcke, J. Phys. Chem. A 110, 13238 (2006).

${ }^{72}$ M. Merchán, R. González-Luque, T. Climent, L. Serrano-Andrés, E. Rodríguez, M. Reguero, and D. Peláez, J. Phys. Chem. B 110, 26471 (2006).

${ }^{73}$ G. Zechmann and M. Barbatti, J. Phys. Chem. A 112, 8273 (2008).

${ }^{74} \mathrm{~S}$. Yamazaki and T. Taketsugu, J. Phys. Chem. A 116, 491 (2012).

${ }^{75}$ H. R. Hudock, B. G. Levine, A. L. Thompson, H. Satzger, D. Townsend, N. Gador, S. Ullrich, A. Stolow, and T. J. Martínez, J. Phys. Chem. A 111, 8500 (2007).
${ }^{76}$ D. Asturiol, B. Lasorne, M. A. Robb, and L. Blancafort, J. Phys. Chem. A 113, 10211 (2009).

${ }^{77}$ J. J. Szymczak, M. Barbatti, J. T. Soo Hoo, J. A. Adkins, T. L. Windus, D. Nachtigallová, and H. Lischka, J. Phys. Chem. A 113, 12686 (2009).

${ }^{78}$ Z. Lan, E. Fabiano, and W. Thiel, J. Phys. Chem. B 113, 3548 (2009)

${ }^{79}$ H. Kang, K. T. Lee, B. Jung, Y. J. Ko, and S. K. Kim, J. Am. Chem. Soc. 124, 12958 (2002)

${ }^{80}$ Y. He, C. Wu, and W. Kong, J. Phys. Chem. A 107, 5145 (2003).

${ }^{81}$ Y. He, C. Wu, and W. Kong, J. Phys. Chem. A 108, 943 (2004).

${ }^{82}$ S. Ullrich, T. Schultz, M. Z. Zgierski, and A. Stolow, Phys. Chem. Chem. Phys. 6, 2796 (2004).

${ }^{83}$ C. Canuel, M. Mons, F. Piuzzi, B. Tardivel, I. Dimicoli, and M. Elhanine, J. Chem. Phys. 122, 074316 (2005).

${ }^{84}$ P. M. Hare, C. E. Crespo-Hernández, and B. Kohler, Proc. Natl. Acad. Sci. U.S.A. 104, 435 (2007).

${ }^{85}$ R. Abouaf, J. Pommier, and H. Dunet, Chem. Phys. Lett. 381, 486 (2003).

${ }^{86}$ T. Gustavsson, Á. Bányász, E. Lazzarotto, D. Markovitsi, G. Scalmani, M. J. Frisch, V. Barone, and R. Improta, J. Am. Chem. Soc. 128, 607 (2006).

${ }^{87}$ T. Gustavsson, Á. Bányász, N. Sarkar, D. Markovitsi, and R. Improta, Chem. Phys. 350, 186 (2008).

${ }^{88}$ A. Nakayama, G. Arai, S. Yamazaki, and T. Taketsugu, J. Chem. Phys. 139, 214304 (2013). 